\title{
FEDERAL MOTOR CARRIER SAFETY ADMINISTRATION'S RESEARCH AND TECHNOLOGY INITIATIVES TO ENHANCE COMMERCIAL DRIVER TRAINING, LICENSING AND PERFORMANCE MANAGEMENT
}

\author{
Jerry Robin \\ FMCSA Research Division \\ Department of Transportation \\ Federal Motor Carrier Safety Administration \\ 400 Virginia Avenue, S.W. (MC-RTR) \\ Washington, DC 20024 \\ E-mail: Jerry.Robin@fhwa.dot.gov \\ Ronald Knipling \\ FMCSA Research Division \\ Department of Transportation \\ Federal Motor Carrier Safety Administration \\ 400 Seventh Street, S.W. (MC-RTR) \\ Washington, DC 20590 \\ E-mail: Ron.Knipling@fhwa.dot.gov
}

\begin{abstract}
Summary: This paper discusses several of the numerous commercial motor vehicle (CMV) driver training and safety performance enhancement initiatives being conducted by the Federal Motor Carrier Safety Administration (FMCSA), Office of Research \& Technology (R\&T). Programs discussed include the Truck Simulator Validation Study, the Pilot Test of Fatigue Management Technologies and the Intelligent Vehicle Initiative: Heavy Vehicle Platform - Generation Zero Operational Tests. The document will also serve to acquaint the reader with the mission of the FMCSA. Additionally, it provides an overview of the vast scope of research being conducted by the FMCSA R\&T Office.
\end{abstract}

\section{INTRODUCTION}

The Federal Motor Carrier Safety Administration (FMCSA; formerly the Federal Highway Administration Office of Motor Carriers [FHWA OMC]) is a new agency established on January 1, 2000 within the U.S. Department of Transportation. Its mission is to improve truck and bus safety on our Nation=s highways. The goal of FMCSA is to reduce the CMV related fatalities and injuries from crashes by 50 percent by the year 2010. Using 1998 as baseline year, FMCSA intends to reduce fatalities from 5,374 to 2,687 (or less) and reduce the number of injuries from 127,000 to 63,500 (or less) by 2010 (FARS 1998, GES, 1998). Meeting these aggressive goals will require many concerted and innovative motor carrier safety activities involving government, industry, and, most importantly, drivers.

The FMCSA Office of R\&T is responsible for planning, administering, coordinating, and managing agency R\&T to support FMCSA's regulatory and other operational programs. The Program seeks to gain fundamental and applied knowledge, and to develop new methods and technologies to enhance truck and bus safety. The FMCSA R\&T program is segmented into 5 
categories, as follows:

1. Driver Safety Performance

2. Carrier Safety and Compliance

3. Vehicle Safety Performance

4. Safety Systems and Technologies

5. Cross-Cutting Safety Initiatives

Driver Safety Performance ensures that commercial drivers are physically qualified, trained to perform safely, and mentally alert. This research area also helps improve the safety behavior of non-commercial drivers in the vicinity of trucks and buses. It includes four subcategories: CMV Driver Training, Licensing, and Performance Enhancement; CMV Driver Fatigue; CMV Driver Physical Qualifications; and Non-CMV Driver Behaviors.

Carrier Safety and Compliance improves motor carrier compliance with Federal Motor Carrier Safety Regulations and their safety practices in general.

Vehicle Safety Performance improves truck and bus performance through the adoption of vehicle-based safety technologies. The major area under this category is the commercial (heavy) vehicle platform of the Intelligent Vehicle Initiative (IVI) which is managed by FMCSA and coordinated with NHTSA.

Safety Systems and Technologies initiatives identify, test, evaluate, and deploy new technologies and operational concepts that improve CMV safety; and target high-risk carriers for enforcement and compliance actions.

Cross-Cutting Safety initiatives allow FMCSA to gain a deeper and broader understanding of driver, carrier, vehicle, and roadway environmental factors that place CMVs at risk, and the potential applicability of countermeasures. This category also enhances the management, effectiveness, and impact of the overall agency R\&T program.

The specific programs discussed in this paper include the following:

- $\quad$ Truck Simulator Validation Study (“Sim Val”)

- $\quad$ FMCSA National Advanced Driving Simulator (NADS) Utilization Study

- $\quad$ Pilot Test of Fatigue Management Technology

- $\quad$ CMV Driver Assessment Tool

- $\quad$ Driver Retention and Safety

- Driver Health and Wellness Program

- $\quad$ Intelligent Vehicle Initiative: Generation Zero Operational Tests

- $\quad$ Potential future research to improve CMV driver training and performance

This paper draws heavily from various FMCSA internal documents maintained by the authors and other internal documents. A discussion of the importance of the human factor in crash reduction follows. 


\section{THE DRIVER AND TRUCK-INVOLVED CRASHES}

Commercial drivers are generally good drivers. CMV crashes, like motor vehicle crashes in general, are caused principally by human error and/or misbehavior. The crash involvement rate per mile traveled of combination-unit truck (tractor-semitrailer) drivers is less than one-half that of non-commercial drivers, and a much smaller percentage of their crash involvements are as the “at fault” vehicle (Wang, Knipling, and Blincoe, 1999). The majority of car-truck crashes are related more to the errors and misbehaviors of car drivers than to those of truck drivers (FARS 1998). Moreover, commercial drivers are less likely than non-commercial drivers to seriously violate speed limits (NHTSA, 1991) or engage in aggressive or risky driving behaviors. Of fatal truck-passenger vehicle crashes from 1994 to 1998, 38 percent were found to have a contributing factor relating to the commercial driver and 65 percent a contributing factor relating to a noncommercial driver or other road user (Craft, 2000). However, because of the high mileage exposure of trucks and the oftentimes severe consequences of their crashes, there is a premium on making trucks, and truck drivers, safer. Annual crash costs are more than four times greater for a combination-unit truck (tractor-trailer) than for a passenger car (Wang, Knipling, and Blincoe, 1999). The importance of truck safety is highlighted by the fact that approximately about $75 \%$ of all injuries from combination-unit truck crashes, and approximately $86 \%$ of fatalities, occur "outside" of the truck; i.e., to other motor vehicle occupants and non-motorist involved in crashes with combination-unit trucks (GES, 1999).

Each of the aforementioned R\&T programs will now be discussed.

\section{TRUCK DRIVING SIMULATOR VALIDATION (“SIM VAL”) STUDY}

Simulators have been successfully employed within the military and commercial sectors for several decades. Now relatively low-cost, full-mission, high-fidelity CMV simulators are available in the marketplace and being employed by various organizations in the trucking industry. As the cost of these devices continue to decrease, while the capabilities seemingly increase, their use will likely increase as well. Truck simulators may be useful tools to supplement training, testing and licensing of CMV operators. However, there has been little, if any, previous effort by either the private or public sector, to empirically examine the transferability of simulation training to real driving, or to assess its usefulness, effectiveness, or efficiency (Emery, Robin, Knipling, Finn, and Fleger 1999).

One of the primary objectives of the "Sim Val" study is to examine how simulation-based training, as compared to conventional training methods, can enhance the training environment and improve the tractor-trailer driver performance. The study will also identify opportunities employing truck driver training simulation technology and examine cost implications.

The Sim Val study is culmination of a number of interrelated research activities conducted by FMCSA over the past several years. The initial truck simulator assessment determined that truck simulator technology was sufficiently mature to conduct a validation study (FHWA, 1996). FMCSA then developed and published a detailed research design and the plan to conduct the empirical Sim Val study (Emery, Robin, Knipling, Finn, and Fleger 1999). A second, more detailed, truck simulator re-assessment, is currently being conducted by Veridian Engineering. It is 
based on the simulator functionalities described in the research design report. The completion of the re-assessment report will assure that FMCSA has the most up-to-date information on commercially available truck simulators in the U.S. The re-assessment report is scheduled to be complete by September 2001. A request for proposals for the actual Sim Val study will be made in FY’02.

The research design reflects the input of industry based on two peer reviews; one on the driving scenarios to be used in the study and a second on the research design itself. It consists of three parts, as follows:

Part 1 - Addresses the forward transfer of training (conventional tractor-trailer vs. simulator -based training) for entry-level drivers. It includes a pilot test.

Part 2 - Assessment of advanced capabilities (emergency maneuvers, front tire blow out, etc.) of the test simulator. It includes a pilot test.

Part 3 - Longitudinal study of drivers who have successfully completed Part 1 and gone on to earn their Commercial Drivers License. Retrospective assessments of driver safety performance will be conducted at the 3-month and 12-month marks following the CDL test.

\section{NATIONAL ADVANCED DRIVING SIMULATOR (NADS) UTILIZATION STUDY}

TheNADS, a \$60 million state-of the-art simulator, under the stewardship of the National Highway Traffic Safety Administration (NHTSA), will be used to conduct fundamental research in highway safety, integrated vehicle highway systems and advanced vehicle design. It is located at the University of Iowa and is currently operational. FMCSA engaged Veridian Engineering to develop a FMCSA NADS Utilization Report. The report will assess NADS capabilities from a FMCSA truck perspective and result in a number of potential NADS projects that can be selected from during the next few years. Brainstorming sessions have been conducted at FMCSA to help generate candidate projects and to develop a process for successfully integrating the NADS into FMCSA R\&T Program. A test drive of the NADS configured with the truck buck is also planned so as to better understand the capabilities of this device. FMCSA regards NADS as a useful research tool for addressing many driver-related human factor issues including driver fatigue, medical conditions, driver selection and training (not as a driver trainer per se, but as a tool to improve training simulators and methods), human performance evaluation/enhancement and technology assessment for improving CMV driver safety. However, like any research tool, its applicability and cost/benefit effectiveness for any research problem will be assessed.

\section{DRIVER ASSESSMENT TOOL}

This project, being procured as a Small Business Innovation Research (SBIR) Phase I proof-ofconcept study will demonstrate the feasibility and validity of a fast, low-cost, portable assessment tool to measure basic CMV cognitive skills and behaviors relevant to safe CMV driving. The Volpe Center administers the SBIR Program for DOT. The purpose of the SBIR program is to invite small businesses to submit innovative research proposals that address high organizational priorities. 
An emerging trend in human resource management in the CMV industry is likely to be increasing sophistication and reliance on technology, such as the use of validated selection methods, driving simulators for training, and on-board monitors for performance evaluation. Applied research will develop and assess such approaches and prove (or disprove) that they enhance CMV driver performance and safety. This project focuses on the driver selection and performance evaluation/enhancement components of CMV driver human resource management. Critical to improving driver selection and performance assessment is the development of more valid and predictive selection tools, including personal history inventories, psychological tests, and psychomotor tests. For example, a 30- to 45-minute PC-based simulation might provide drivers with traffic situations calling for safe vehicle control, defensive driving, crash threat recognition, reaction to threat, decision-making, and execution of evasive maneuvers. The envisioned tool would not be a high-fidelity simulation of the physical driving environment, but rather would focus on assessing cognitive/behavioral performance traits and behaviors relevant to safe CMV driving. This project will support the validation and development of such tools. The principal applications of this device would be for motor carriers to improve their CMV driver selection processes and the safety performance assessment and improvement of current drivers. The Phase 1 study will commence in FY'02. If successful and promising, the Phase I proof-of-concept phase could be followed by Phase II research application and product development.

\section{DRIVER HEALTH AND WELLNESS}

Truck driving, particularly long haul truck driving, is largely sedentary in nature, with few opportunities for drivers to exercise. Exercise has been shown to combat fatigue, reduce stress, improve alertness and enhance sleep. As a result, the trucker who exercises will be better rested and more alert for driving. Yet, the literature indicates that a larger percentage of truck drivers tend to be overweight, smoke, eat less healthy, and exercise less, as compared to the general population (Robin and Roberts, 1999)

To help raise driver and Industry awareness of the importance physical fitness and other healthy lifestyle choices, FMCSA worked collaboratively with National Private Truck Council, American Trucking Associations Foundation Trucking Research Institute (ATA/TRI) and Sue Roberts Health Concepts, Inc., to develop a truck driver health and wellness program. The program, called “Getting' In Gear,” is comprised of four components: 1. Refueling (eating well) 2. Relating (interpersonal skills) 3. Relaxing (stress management) and 4. Rejuvenating (health and fitness). The program also employs an attitude behavior change model which conceptualized four stages in the path toward positive change: precontemplating/denial, contemplation, preparation/action and maintenance. This program was pilot-tested at about six locations throughout the U.S. and revised to reflect lessons learned. The “Getting' In Gear” Kit includes videos, workbooks and a train-the trainer component. The Kits will be available in Summer 2001.

\section{FATIGUE MANAGEMENT TECHNOLOGIES PILOT TEST}

FMCSA is sponsoring a Congressionally-directed pilot test to demonstrate the use of fatiguerelated technologies to enhance CMV driver fatigue management. The ATA/TRI is the principal project contractor to FMCSA. Participants also include Transport Canada, University of 
Pennsylvania, Carnegie Mellon Research Institute, Walter Reed Army Institute of Research, Assistware, Inc., and River City Products.

The Fatigue Management Pilot Test (FMT) combines driver alertness monitoring (using the eyelid droop measure known as PERCLOS), already a major technological component of the ITS Intelligent Vehicle Initiative (IVI), with other fatigue management technologies. These other technologies include a wrist-worn sleep monitor (actigraph) and sleep-wakefulness models, a lane tracking device (SafeTRAC), and the Howard Powered Center Steering System, a fatigue reducing, automatic steering Acentering@ system. The actigraph, PERCLOS monitor, and lane tracker will all provide direct feedback to drivers on their amount of sleep, readiness, "eye open” alertness and lane keeping performance. The lane tracker will also be used to provide lane departure warning to drivers. Numerous research studies sponsored by the U.S. DOT and others have provided proof-of-concept for the validity of the various alertness/ performance measurements employed. These technologies are now ready to progress from laboratory/experimental use to use as fatigue management aids in an operational "Beta-Test." The FMT Pilot Test includes 2 Phases.

Phase 1 assessed available technology and developed a detailed research design and plan. A "Pre-Pilot” over-the-road test of equipment suite was successfully completed in 2000.

The Phase 2 study will be conducted separately in the U.S. and Canada under current HOS rules. Pilot data collection will begin in Canada, involving Challenger Motor Freight. U.S. fleets will be brought on board shortly thereafter. There will be baseline and post-intervention gathering of data on driver alertness, performance, and individual differences; and the use of concurrent validated tests of driver alertness such as the psychomotor vigilance test (PVT). In addition, Phase 2 will establish an alertness baseline for each driver, measure the reliability of individual differences in alertness across time, and determine the validity of various measures to assess susceptibility to fatigue. In the experimental (operational test) phase, drivers and their managers will be taught to use the technological devices as fatigue management aids. Before and after measures of driving performance and safety will be compared and the practical benefits of technological fatigue countermeasures will be assessed. Data collection will start in Fall 2001 and the project is scheduled for completion in late 2002.

The principal hypotheses are that the use of these fatigue management technologies will encourage drivers to practice sleep hygiene (i.e. nighttime sleep and stop for naps when driving) and will be reflected in higher level of alertness and performance while driving. FCMSA believes that fatigue management technologies should be viewed primarily as aids to self and fleet management, not as instruments of surveillance.

\section{DRIVER RETENTION AND SAFETY}

Annual driver turnover rates of $100 \%$ are common in for-hire trucking, especially the long-haul truckload segment. Industry-wide, fleets with low driver turnover rates are generally characterized by high safety standards and performance. It appears that one avenue to improved driver and fleet safety is to improve retention. Fleets with high driver turnover rates perhaps lower their selection standards for new hires and expedite orientation and training for new hires 
to meet their operational needs for drivers. Such fleets may be unable to develop and implement safety improvements because of their preoccupation with filling quantitative staffing needs. In fleets with good driver retention, it appears that drivers are able to acclimate to their jobs, routes, and work patterns, with resulting decreases in incidents and crashes, although empirical documentation of this acclimation process is not strong. One important question relates to the causal basis for the relation between fleet turnover rates and safety; does this simply reflect better selection of drivers or is there demonstrable improvement of safety for individual drivers associated with acclimation to a particular job?

A study, which will commence in Summer 2001, will begin to answer this question by reviewing the literature linking driver retention and safety and summarize carrier practices to improve driver retention. The project will also recommend both research activities and industry-directed safety interventions to address this problem. FMCSA engaged TransAnalytics, LLC to conduct this 12-month study.

\section{INTELLIGENT VEHICLE INITIATIVE: HEAVY VEHICLE PLATFORM - GENERATION ZERO OPERATIONAL TESTS}

The Intelligent Vehicle Initiative (IVI): Heavy Vehicle Platform is part of a larger DOT program called the Intelligent Transportation System (ITS). The IVI has four platforms for technology development and testing: light vehicle, transit vehicle, heavy vehicle, and specialty vehicle. FMCSA manages the heavy vehicle platform which involves CMVs: trucks, tractor-trailer combinations and, interstate buses.

The primary goal of the IVI is to accelerate development, introduction and commercialization of vehicle-based, driver assistance products to reduce crashes. Rear-end, lane-change and roadway departure crash avoidance systems have collective potential to reduce motor vehicle crashes by 1.2 million annually based on preliminary NHTSA estimates. The IVI Generation Zero ("Gen Zero”) field tests are testing several different safety technologies in commercial vehicle fleets. This project focuses on vehicle-based driver assistance products that will be commercially available with the next five years. It is important to determine if these systems will have a positive or negative impact on safety and performance, and how multiple systems will impact driver performance in actual commercial service. Data collection will be conducted during FY'01 and FY'02 with all work complete by late 2002. Currently, FMCSA is conducting three "Gen Zero" operational tests.

The first test is an assessment of the collision warning system (advanced Eaton-Vorad) including closing distance warning, blind spot object warning, and adaptive cruise control will be evaluated on 50 heavy vehicles with an additional 50 vehicles being used as the control group.

The second Gen Zero test is the infrastructure-assisted road hazard warning system which will be evaluated on 143 commercial tractors based in Virginia.

The third involves a truck Rollover Stability Advisor (RSA) to warn truck drivers of potential instability. Six tractors coupled to tank semi-trailer will operate in the Midwest. Three of the tractors will be equipped with the countermeasure and three will serve as the unequipped control group. 
Late 2002, FMCSA plans to begin IVI operational tests of electronically-controlled braking systems that will reduce stopping distance and improve vehicle stability, drowsy driver detection and warning device to reduce inattention and fatigue crashes; and rear impact warning devises mounted on the back of commercial vehicles to alert following vehicles.

\section{POTENTIAL FUTURE RESEARCH INITIATIVES TO IMPROVE CMV DRIVER TRAINING AND PERFORMANCE}

In the coming years, FMCSA may explore the use of simulators and other technologies to enhance the commercial drivers license program. In addition, research will be conducted to define the most important performance and behavioral measures of CMV driving quality. Impaired driving and the validation of safety technologies are additional areas of organizational interest. To complement FMCSA “No Zone” activities, interventions will be developed, including educational and technologies, to prevent "car-truck proximity" crashes, both the large number precipitated by non-CMV driver and those precipitated by the CVM drivers themselves.

\section{CONCLUSION}

Human factors research sponsored by the FMCSA employs a variety of research methodologies, including on-road in-vehicle observations, driving simulator, laboratory studies, expert panels, surveys, and crash data analysis. Enhanced management of driver performance and improved training, testing, licensing methods are being achieved through the assessment and deployment of potentially life saving technologies, reducing the potential for crashes. In addition, new management practices for training and driver evaluation are being developed to instill safety awareness into everyday fleet management. Research to identify, evaluate, and select recommended management practices for modifying driver behavior and improving the performance, particularly of high-risk drivers, will result in reduced traffic violations, injuries and fatalities.

\section{REFERENCES}

Craft, R., The Large Truck Crash Picture, FMCSA, Office of Data Analysis and Information Systems, August, 2000.

Emery, C., Robin, J., Knipling, R., Finn, R., and Fleger, S., Research Design: Validation of Simulation Technology in the Training, Testing, and Licensing of Tractor-Trailer Drivers, FHWA Final Report FHWA-MC-99-060, June, 1999.

FHWA, Commercial Motor Vehicle Simulation to Improve Driver Training, Testing and Licensing Methods (FHWA-MC-96-003), April, 1996.

NHTSA, Fatality Analysis Reporting System (FARS), 1998.

NHTSA, General Estimates System (GES), 1998, 1999. 
NHTSA, Commercial Motor Vehicle Speed Control Devices. Report to Congress. Publication No. DOT HS 807 725, NHTSA Office of Crash Avoidance Research, 1991.

Robin, J., Roberts, S., Truck Stop Fitness Facility Utilization Study, Proceedings from the Second International Truck and Bus Safety Symposium, Transportation Center, University of Tennessee, October, 1999.

Wang, J.S., Knipling, R.R., and Blincoe, L.J. The dimensions of motor vehicle crash risk. Journal of Transportation and Statistics. Volume 2, Number 1, Pp. 19-43, ISSN 1094-8848, May, 1999. 